Fukunaga et al., 2019

Volume 3 Issue 1, pp. 88-97

Date of Publication: 15 $5^{\text {th }}$ March 2019

DOI-https://dx.doi.org/10.20319/pijtel.2019.31.8897

This paper can be cited as: Fukunaga, R., Sasaki, H. E Hanis, I. B. M., (2019). How to Evaluate a Program Written by Children: A Program Evaluation Support Tool for Elementary School Teachers. PUPIL: International Journal of Teaching, Education and Learning, 3(1), 88-97.

This work is licensed under the Creative Commons Attribution-Non Commercial 4.0 International License. To view a copy of this license, visit http://creativecommons.org/licenses/by-nc/4.0/ or send a letter to Creative Commons, PO Box 1866, Mountain View, CA 94042, USA.

\title{
HOW TO EVALUATE A PROGRAM WRITTEN BY CHILDREN: A PROGRAM EVALUATION SUPPORT TOOL FOR ELEMENTARY SCHOOL TEACHERS
}

\author{
Rie Fukunaga \\ Faculty of Engineering, Takushoku University, Tokyo, Japan \\ rfkng@eitl.cs.takushoku-u.ac.jp \\ Hitoshi Sasaki \\ Faculty of Engineering, Takushoku University, Tokyo, Japan \\ sasaki@cs.takushoku-u.ac.jp \\ Ilyani Binti Mazlan Nur Hanis \\ Faculty of Engineering, Takushoku University, Tokyo, Japan \\ nuhail@eitl.cs.takushoku-u.ac.jp
}

\begin{abstract}
Programming education will be an obligation at primary school in Japan starting in 2020. This leads elementary school teachers evaluate programs that are created by the students. However, a clear standard of evaluation which is necessary for teachers as an evaluation guidance has not been stipulated yet. The programming obligation will surely give a big burden to the them. Therefore, we are developing an application that support program evaluation, which can support teachers in evaluating programs created by children and enable to connect with the future guidance.

A Program created by the children using Scratch will be read as an image and analyzed according to the evaluation item using template matching. By presenting materials to support evaluation using an application to the teachers, it can reduce their burden. In addition, by
\end{abstract}


converting the results into data, it is easier to judge whether or not the children understand the content of the lesson, the teachers can respond to the children who cannot follow the class at an early stage.

\section{Keywords}

Programming Education, Primary School in Japan, Program Evaluation

\section{Introduction}

\subsection{Current Status of Programming Education in Japan}

In Japan, programming education at primary school will be an obligation starting in 2020 . Today, computer is used in various places. In order to handle it properly, having programming thought and knowledge are important. Besides, learning about these will help extend the possibilities of children. There is also a move to introduce programming education from primary education abroad (Go Ota et al., 2016) . In the future, it is expected that mechanization will advance, thus, there is a concern on shortage of human resources in IT industry. Even now, there is a shortage of human resources in IT, and it proves that training for human resources is necessary (Ministry of Education, Culture, Sports, Science and Technology, 2018).

Elementary school teachers will evaluate program created by children, but then, the clear standard of the evaluation which is necessary to use for teachers' guidance, is not yet stipulated. In addition, many of the teachers are concern whether they can evaluate the program adequately or not since most of them themselves are inexperienced in programming. Despite activities such as opening a programming course for teachers are done according to region (Nikkei Business Publications, Inc. 2018). This research stated that it is difficult to provide proper guidance to all teachers at the moment. From the program created by students, the teachers have to guide them from their tenacious, scheming, and weak points that are found. Therefore, we are developing an application that support program evaluation, which can support teachers in evaluating programs created by children and enable to connect with the future guidance.

\subsection{Visual Programming}

In the material published by Ministry of Education, Culture, Sports, Science and Technology (MEXT), visual programming is mentioned as a specific educational method (Ministry of Education, Culture, Sports, Science and Technology, n.d.). As a visual programming language learning environment, there are programming developed by MEXT (Ministry of Education, Culture, Sports, Science and Technology, n.d.), VISCUIT (Digitalpocket c2018) 
developed by NTT and so on. This application covers for Scratch program which is cited in many books as an example in programming education for elementary school.

Scratch (MIT Media Laboratory c2019) is a one of visual programming language learning environment developed by MIT Media Lab in 2006 and is used by more than 20 million people in more than 150 countries. In the Scratch 2.0, by combining 12 types and 144 blocks, a visual program can be created without even using a code as shown in Figure 1. Created program also can be shared with other users.

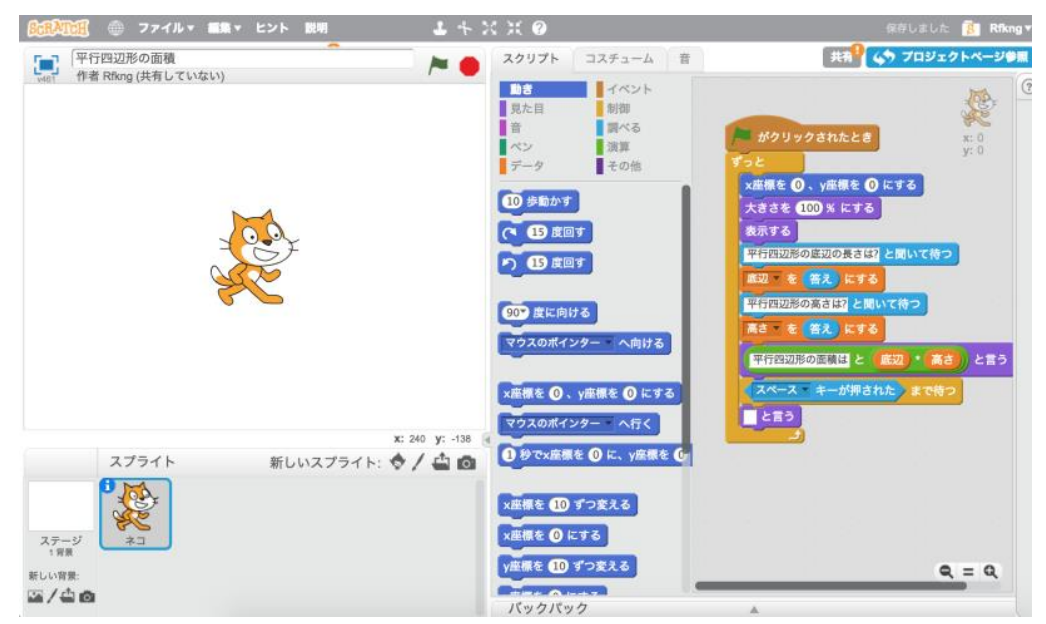

Figure 1: An Example of Scratch Program for Calculating the Area of Parallelogram

\section{Current Status of Programming Evaluation}

Currently, the curriculum is examined and created, and after a demonstration class at a model school is conducted, compulsory programming education will begin. Some local government have already started programming education at elementary schools such as in Tsukuba City in Ibaraki Prefecture, and Kashiwa City in Chiba Prefecture (Eri Ono \& Hitoshi Susono 2017). In the curriculum of programming learning and documents of teaching plan, the curriculum and lecture contents are stated but the evaluation standard is not clarified (Tsukuba General Educations Lab. 2018).

In the ScratchED (ScratchEd 2019) online community educator, several program evaluation sheets have been posted, but all of them required teachers' programming skill. Kashiwa City's Board of Education points out that since it is hard for busy teachers to acquire new programming skill, it is necessary to have a mechanism that enable classes to be conducted even though they cannot program (The Mainichi Newspapers 2018). 
In a research on the evaluation method of the programming project using Scratch conducted by Faculty of Education in Mie University (Eri Ono \& Hitoshi Susono 2017), two rubrics are given. In one of them, the program created by children is evaluated in form of a sheet according to 4 points of view as Table 1 .

Table 1: Evaluate Points

\begin{tabular}{|l|l|}
\hline \multicolumn{1}{|c|}{ Point of view } & \multicolumn{1}{c|}{ Detail } \\
\hline Knowledge of subject & Understanding on what they learned in the subject \\
\hline Project design & Existence of originality from the design point of view \\
\hline Programming knowledge & $\begin{array}{l}\text { Understanding how the blocks work and able to create logical } \\
\text { combination }\end{array}$ \\
\hline Learning process & $\begin{array}{l}\text { Accurately utilizing the basic process and progressing in good } \\
\text { operation }\end{array}$ \\
\hline
\end{tabular}

These viewpoints are currently designed to be used in Japanese education sites with references to the rubric which is the criterion for evaluating the scratch project posted on ScratchEd.

\section{Point of View in Program Evaluation}

\subsection{Outline of Point of View in Evaluation}

Everyone's program will be evaluated by guidance in form of class or group. Besides, since programming classes will not be created but instead programming elements will be introduced in existing classes, it is necessary to examine the contents of the classes. In addition to this, since there is a need for the teachers themselves to acquire programming knowledge, the programming obligation will surely give a big burden to the them.

By presenting materials to support evaluation using an application to the teachers, it can reduce their burden. Also, converting the result to data makes it easier to judge whether you understand the content of the lesson. In addition, teachers will be able to respond to children who could not follow during class hours. At the same time, it is easy to compare the average of class or group with the previous evaluation result. By referring to the results for each evaluation item, the labor for confirming each block used in the program created by the children is reduced, thus, providing a support for final evaluation. 
The following stage are calculated for the evaluation points from a Scratch program written by a child. The score of each stage is evaluated in five viewpoints.

1. Earnestness: Number of Blocks Arranged Vertically

2. Control \& Structure: Number of Conditions used in Blocks

3. Idea Implementing: Number of Variables, List, and Definition Blocks

4. Screen Changing and Media Utilization: Number of Operating Blocks and Sound Blocks

5. Subroutine: Total number of Blocks

\subsection{Stage 1: Earnestness}

The program gets longer vertically if lot of blocks are used. Based on that, since combining a lot of blocks lead to the uniqueness of the program, from here we can evaluate the children's effort and how much they actually give initiative to the class. By this, it is evaluated. However, since there will be cases such as the children able to combine the block suitably without understanding how the block works, or is it a meaningful combination or not, thus, we cannot evaluate it by their earnestness only.

\subsection{Stage 2: Control Structure}

If the children manage to use nest and conditional blocks correctly, we can evaluate whether the children can understand the basic of how the block works or not. This stage is evaluated by looking at nest and conditional blocks that are frequently used even for samples with relatively low difficulty such as making the character on the screen moves or drawing figures. Many of these programs can be created without using nests or conditional blocks. In that case, the evaluation of the earnestness is high, and the evaluation of the control structure is low. By comparing the evaluation of the earnestness and the control structure, we can respond to the children who cannot follow the class at an early stage.

\subsection{Stage 3: Idea Implementing}

We thought that we can evaluate the children's understanding on how the block works if the students are able to use variables and list blocks created by themselves properly. Evaluating this stage will be done by the usage of variables and list blocks in samples with relatively high difficulty, such as manipulating character on the screen according to input and creating games such as quizzes and so on. Children who use variables and list blocks correctly and who are highly evaluated in the implementation of ideas are deemed to have high programming abilities. 


\subsection{Stage 4: Screen Changing and Media Utilization}

Most blocks in Scratch are motion and sound related blocks. If these blocks are used a lot, we can evaluate that the children are interested in these blocks and have a curiosity in trying them. Furthermore, since the earnestness stated in stage 1 (Earnestness) itself is not enough to be evaluated in the uniqueness of a program, their effort will be evaluated with this stage.

\subsection{Stage 5: Subroutine}

The easiest way to compare sample code, which is a sample answer, is by the number of blocks used in a program. Even when the target result can be produced but the number of blocks is lesser or more than the sample code, both cases will relate with the final evaluation. The above point is evaluated under stage 5 .

\subsection{Comparison and Usage with Previous Research}

Next, from the viewpoint of the previous study, "Project Design" and "Programming Knowledge" in Table 1, that have a direct relation to program are compared to evaluation item in this research and will be examined whether it will become the material for final evaluation.

"Project Design" is evaluated by three points as stated below.

1. Originality of the product

2. Organization of the contents

3. Is it a method for other children to interact with the product?

In this research, from the evaluation item in stage 4 (Screen Changing and Media Utilization), evaluation that is connected to the final evaluation in "Project Design" is done by evaluating from the occurrence of an event, the movement and change of a sprite and the number of blocks related to BGM and sound effects.

"Programming Knowledge" is evaluated by three points as stated below.

1. Understanding the function of the blocks and their usage or not

2. Logically made or not

3. Containing error or not

If blocks related to condition and variables are used in the program, and the output shows the correct result, we can say that they understand the blocks' function and are logically created. 
Since the program does not operate correctly when there is an error, the third point will not be considered in this research.

From the evaluation items in this research which are stage 2 (Control Structure) and stage 3 (Idea Implementing), evaluation will be shown by evaluating from the number of blocks related to condition and variables, which leads to final evaluation of "Programming Knowledge".

\section{Evaluation Application}

\subsection{Outline of Application}

Program created by the children using Scratch will be read as an image and analyzed according to the evaluation item using template matching. The image of each block will be the original image, and from the program created by the children, the number and arrangement of each block is detected. This system makes evaluates every stage by using these results. From the result of evaluations, as shown in Figure 2, a graph is drawn in the output screen plan along with visually shown evaluation results. On the left side of the screen is an imported program list of Scratch, on the center of the screen are the program evaluation from this time and last time, and on the right side of the screen are individual evaluation results along with a graph that shows evaluation results up until present. By outputting two graphs, it is easier to judge the children's learning progress and their special fields.

As mentioned in the outline of the evaluation point of view, teachers will guide them in form of class or group. By visually outputting the result with a radar chart as shown in Figure 3, it can be compared easily rather than only outputting letters. This makes it easier for evaluation on children's effort, strong points, or fail points.

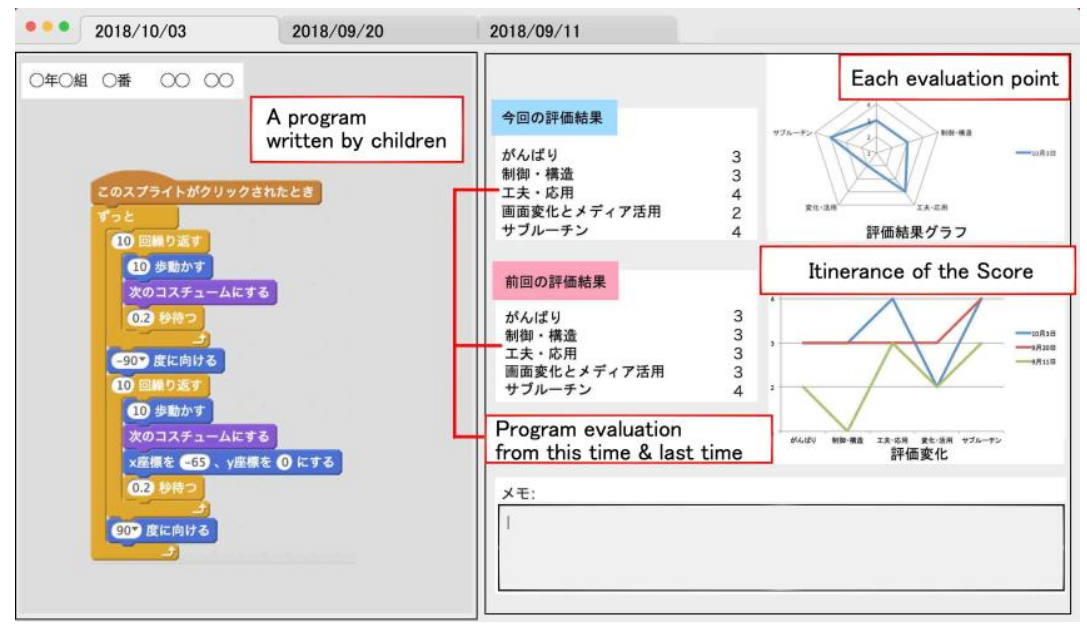

Figure 2: An Example of Analysis Result 


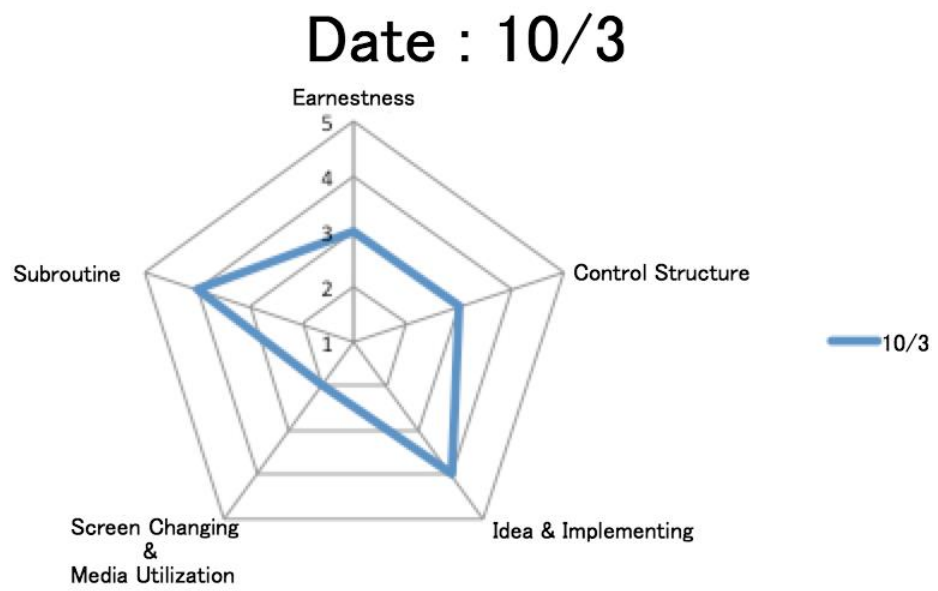

Figure 3: An Example of Evaluation Chart for Each Stage

In addition to the radar chart, by outputting the results with a line graph as shown in Figure 4, comparison with the previous record becomes easy. We can see how much the children have evolved by repeating the number of times. If a high evaluation continues, more advanced guides will be provided, which will be useful for future teaching methods.

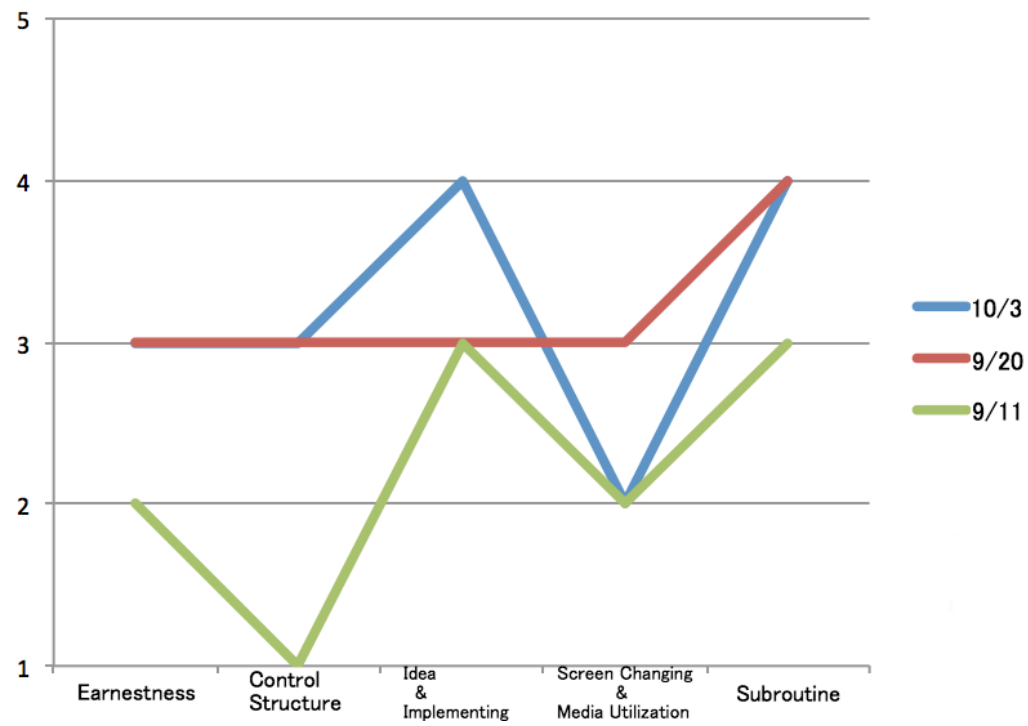

Figure 4: An Example of Time Series Variation

\subsection{Accuracy of Program Analysis}

Using the image of each block of Scratch template, in OpenCV (OpenCV team 2019) template matching (OpenCV dev team 2011), the number and order of each block is detected, and the total result for each type of block is showed as a graph. Simultaneously, evaluation items 
are selected from Scratch's sample code. Furthermore, we compared the selected evaluation items and evaluation viewpoint of the Scratch program evaluation method from the research in Mie University. Currently, variable blocks with user's name on it still cannot be detected. In the future, we are planning to use deep learning to solve this problem. The detection rate of the end of nested block is about $80 \%$ in a sample program that we used.

Whether this recognition rate is sufficient for teacher support or not must be examined in the future. However, in addition to that, we must tackle further improvement in recognition rate. In near future, we are planning to make the nested structure and variable blocks able to judge. And we need reviewing the evaluation items so that they will become easier to understand. Apart from that, we also plan to complete the program, save the result, and create the output screen according to plan.

\section{Conclusion}

We believe that by using the system described in this paper, teachers can give appropriate guidance to children in less time than before. Aiming for an easy-to-understand, easy-to-use application even for instructor who majored in literature, burden of teachers can be reduced by using this application. Also, with the children able to receive appropriate guidance and evaluation, opportunity for them to proceed to IT field can be created. Furthermore, the results of this research can be applied not only to primary education but also to general information education in higher education (Shigeto Okabe 2016).

However, practical studies of programming education using this system have not been done yet. Through these practices, it is necessary to firmly consider whether evaluation criteria are adequate or appropriate.

\section{References}

Digitalpocket. (c2018). Viscuit. Retrieved 17 February, 2019, from http://www.viscuit.com/ Eri Ono \& Hitoshi Susono. (2017). JSSE Research Report. How to evaluate students' Scratch projects in Japan (Vol.31 No.8, pp.107-112).

Go Ota, Yosuke Morimoto and Hiroshi Kato (2016). Japan journal of educational technology, Special Issue: The New Era of Information Education. The Comparative Survey of Computer Science and Programming Education for Primary and Secondary Schools in the UK, Australia and USA Vol. 40, No. 3, 197-208). 
Mit Media Laboratory. (c2019). Scratch - Imagine, Program, Share. Retrieved 17 February, 2019, from https://scratch.mit.edu/

Ministry of Education, Culture, Sports, Science and Technology. (2018). Programming education. Retrieved 17 February, 2019, from http://www.mext.go.jp/a_menu/shotou/zyouhou/detail/1375607.htm

Ministry of Education, Culture, Sports, Science and Technology. (2017). Programin, Retrieved 17 February, 2019, from http://www.mext.go.jp/programin/

Nikkei Business Publications. (2018). Education and ICT Summer issue(pp.7-12).

OpenCV dev team. (2011). Template Matching. Retrieved 17 February, 2019, from https://docs.opencv.org/2.4/doc/tutorials/imgproc/histograms/template_matching/templat e matching.html

OpenCV team. (2019). OpenCV library. Retrieved 17 February, 2019, from https://opencv.org/ ScratchEd. (2019). ScratcheD. Retrieved 17 February, 2019, from http://scratched.gse.harvard.edu/

Shigeto Okabe. (2016). Japan journal of educational technology, Special Issue: The New Era of Information Education. Information Education in Higher Education (Vol. 40, No. 3, pp.143-152).

Tatsuya Horita. (2016). Japan journal of educational technology, Special Issue: The New Era of Information Education. Information Education in Elementary and Secondary Education (Vol. 40, No. 3, pp.131-142).

The Mainichi Newspapers. (2018). Teachers also have no programming experience, They have to prepare for the lesson. Retrieved 17 February, 2019, from https://mainichi.jp/articles/ 20170829/k00/00e/040/186000c

Tsukuba General Educations Lab. (2018). Elementary school programming education guide (2nd edition). Retrieved 17 February, 2019, from https://www.tsukuba.ed.jp/souken/?p=7831 